\title{
HYDROPNEUMATIC ALL-WHEEL SUSPENSIONS: APPLICATIONS, CHALLENGES AND SPECIAL SOLUTIONS
}

\author{
Wolfgang Bauer \\ ARGO-HYTOS GmbH, Industriestrasse 9, 76703 Kraichtal-Menzingen \\ Tel.: +49 7250 76541; E-mail address: w.bauer@argo-hytos.com
}

\begin{abstract}
All-wheel suspensions are generally challenging, particularly if they are equipped with an automatic level control. In mobile machines, the special application conditions make their use even more demanding regarding layout and control. A high center of gravity, load variations, off-road-conditions etc. require special measures to reduce roll and pitch movements. Additionally the uneven ground along with the mechanical suspension setup often create a need to measure and optimize tyre-to-ground forces in order to provide best traction and suspension properties. Hydropneumatic suspensions have all the potential to meet all these requirements and provide a comfortable, safe and efficient suspension setup.
\end{abstract}

Keywords: hydropneumatic, all-wheel, suspension, control

\section{INTRODUCTION}

Hydropneumatic suspensions are well known for their superior qualities in mobile machines: high load capacity, easy and fast level control, adaptable suspension properties, robustness and many more. They provide comfort, safe ride behaviour and efficiency to the machines they are applied to. In many mobile machines, only parts of the under-carriage are actually suspended while others are still rigid or only maybe pivoting. In these cases, normally the front axle is suspended like for example on tractors, dumpers, road sweepers etc.

It is obvious that this partial solution is only a compromise between function, complexity and cost. It comes also with a separation of functions: typically, the rigid axle(s) take(s) over the task to avoid roll movement (=roll stabilization e.g. during cornering) while the suspended axle is responsible for comfort and good traction (=efficiency). In an all-wheel suspension, all these functions are evenly distributed to each of the wheels, which adds complexity to the setup.

\section{WORKING CONDITIONS AND REQUIRE- MENTS IN MOBILE MACHINES}

Usually, mobile machines are used as working machines, while the work consists of transportation of heavy loads or working with (heavy) tools. Therefore it is only natural that the weight on the axles/wheels varies very strongly depending on the working/loading condition sometimes up to ratios above 5:1 (max:min).

With the load or the tool (and their vertical position) comes a change of the height of the center of gravity. However, with increasing load, the COG will also move to a higher position. It is easy to see that this double influence causes a massive change of roll torque during cornering or whilst driving alongside a hill (depending on the working or loading condition). This is a major challenge in the layout of an all-wheel suspension and can best be mastered with a hydropneumatic suspension (see next chapter).

To make the conditions even worse, in many applications the machine needs to have a narrow track width to make it fit to the specific working conditions, for example driving in narrow streets (sweeper) or driving in predefined crop rows (sprayer). The narrow track width worsens the ratio of COG-height to track width and therefore complicates the setting of the suspension parameters and even includes the danger of tipping over sideways.

Mobile machines are frequently used off roads, which is generally quite demanding for suspension systems. Typically the ground is not paved but raw, therefore the surface is rather slippery, particularly when wet. Additionally, off-road surfaces are very uneven, with many random bumps and potholes of different size and 
depth. It also needs to be considered, that off-road machines work in naturally shaped areas with particularly steep surfaces being driven up- and downhill but also alongside hills.

From these above-mentioned adverse working conditions, functional requirements can be deduced for appropriate suspensions (Fig. 1). These requirements are sometimes contradictive, therefore often a suitable compromise needs to be found for the suspension setup.

Slippery conditions require the best possible traction. While traction partly depends on the choice of tyres, inflation pressure, etc. , the other important factor for traction is the tyre-to-ground (short: $\operatorname{ttg}$ ) force. Therefore, it is an absolute must, to distribute the ttg forces of all the wheels as evenly as possible - however limited by the laws of physics, as will be shown later.

In order to cope with the undulations of the ground, a certain minimum suspension travel must be provided. If that's not the case, in extreme conditions at least one of the wheels will lift off the ground $(\rightarrow \operatorname{ttg}$ force $=0$ ) and will therefore loose traction completely. It is recommended to have at least $20 \%$ (better 50\%) more suspension stroke than the highest expected difference of wheel position due to ground unevenness.

The suspension stroke is also connected to the level of comfort offered by the suspension. The more suspension stroke, the softer the suspension can be tuned - however limited by unpleasant drivers sensations like sponginess, loss of controllability or even seasickness. It is recommended for example for a wheel/axle suspension to choose the vertical natural frequency (assumed single mass oscillator) not lower than $1 \mathrm{~Hz}$, maybe only depending on certain conditions (damping, the type of vehicle, actual operation conditions etc.) it is possible to go somewhat below that.

Along with strong load changes (be it due to actual change of payload or due to shifting of weight during cornering/braking/accelerating or hilly driving conditions) and a soft suspension setup come strong position changes. These changes in suspension cylinder length must be compensated quickly and accurately by bringing each cylinder back into its default position. ARGO-HYTOS chose to design a dedicated proportional valve for this purpose, as described in a contribution to the IFK in 2014 [1].

Particularly a high COG and a relatively small track width would allow an undesirable amount of roll movement during cornering - therefore countermeasures need to be taken. A roll compensation can help in such cases if the roll movement is normally rather slow. Roll compensation is basically a quick readjustment of the suspension cylinder length to avoid roll movement. It used for example in the MercedesBenz ABC-suspension setup, which was/is available for example on Mercedes S-class models.

If a roll compensation cannot react quickly enough, for example because of a too quick roll movement or a limited amount of ,levelling power", then also an additional roll stabilization

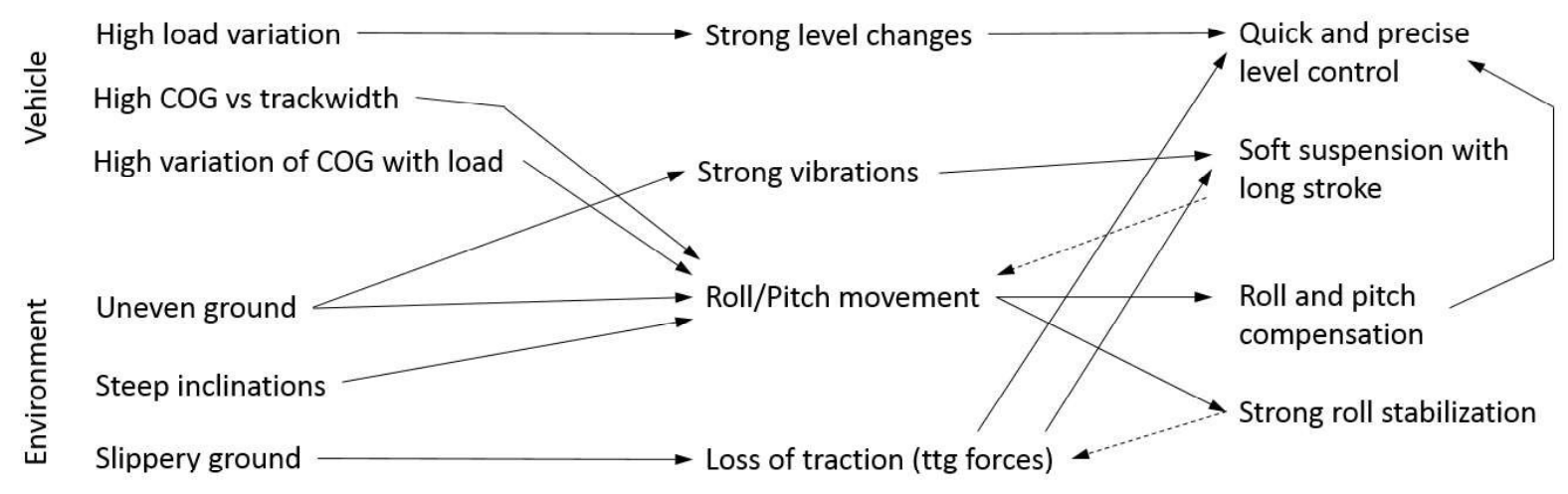

Boundary conditions

Effects

Functional requirements

Figure 1: Boundary conditions, their effects and deduced functional requirements for all-wheel suspensions 
is needed. Roll stabilization comes from an additional spring that connects both wheels of an axle and only creates counterforce, if these two wheels have a different displacement, so when the left wheel is in another position than the right wheel. On passenger cars this is typically done with a stabilizer bar (also referred to as e.g. sway bar or anti-roll bar), which is a torsion bar between both wheels. This can also be found on mobile machines, however if a hydropneumatic suspension is used, the stabilization can also be done purely hydraulically (see next chapter).

Despite of its undeniable positive effect for corner leaning, the roll stabilization also has negative effects on the ride behaviour on uneven surfaces, where the two wheels of an axle ride over different surface profiles. Then the so-called „copy-effect" can spoil the ride behaviour since every acceleration of one wheel is (partly) copied to the other wheel and creates an acceleration there as well. This will create an uncomfortable and harsh ride behaviour with additional accelerations easily visible in the time-positioncurves (also depending on the strength of roll damping). So the amount of roll stabilization must be wisely chosen in order to find the best compromise between reduced roll and acceptable comfort.

\section{SUSPENSION HYDRAULICS: CHALLENGES AND SOLUTIONS}

Hydropneumatic suspensions offer significant advantages for the application in all wheel suspensions in mobile machines. With their additional possibilities however also come certain challenges for the design engineer during the layout of the system. Here are the four most important ones.

\subsection{Accumulator setup}

The accumulator is the component, which determines the spring rate of the suspension. It is the gas inside the accumulator that creates the cushioning effect. So the size and the prefill of the accumulator determine how soft the suspension will feel (the more gas, the softer). As already mentioned before, suspension softness and minimum needed stroke are directly connected to each other. If you allow a stroke of $\pm 100 \mathrm{~mm}$ or more (at the wheel), you can go for a natural frequency of the suspension of 1 to $1,5 \mathrm{~Hz}$ (assumed single mass oscillator). If you only want to only allow $\pm 50 \mathrm{~mm}$ or maybe even less, the natural frequency should be tuned to 2 to $2,5 \mathrm{~Hz}$ (single mass osc.). The aforementioned values of course also depend, among others, upon the chosen damping and the rotational inertia of the chassis. Very generally spoken, significantly below $1 \mathrm{~Hz}$ the suspension will fell too spongy, even uncontrollable, while above 2,5 to $3 \mathrm{~Hz}$ it will not feel comfortable enough.

Typically, diaphragm accumulators are used in hydropneumatic suspensions, sometimes also piston accumulators. The accumulators must withstand all possible pressures that arise from load variations (payload and curve/hillside weight shift) and dynamic pressure changes when riding over uneven terrain. The typical max. pressure ratio of $8: 1$ for a diaphragm accumulator must cope with all these mentioned conditions.

Normally for systems with a load ratio above 2:1 (max:min) a preloaded hydropneumatic suspension is recommended in order to not overstrain the accumulator and to have similar suspension behaviour independent from loading conditions. However, in all-wheel suspensions, oftentimes the roll stabilization is done in a certain way by using the rod chamber of the suspension cylinders, too (shown later in this chapter). Therefore, it is not available to be used for preloading the cylinder hydraulically. Of course, there would be other possibilities of preloading (special cylinder with third chamber or external mechanical preload) but they are complex and costly, therefore a simpler hydraulic solution is preferred.

The rather simple solution consists of an arrangement of two accumulators per cylinder [2]. In such a system, both accumulators are only used with heavy loads while with lower loads, only one accumulator is active. A valve is responsible for switching the second accumulator on and off. The transition point at a medium load is calculated during the layout. During operation, the loading condition is permanently monitored by a measurement of the cylinder pressure (lowpass filtered to neglect pressure variations coming from ground unevenness). If the pressure is above a certain value, the valve is opened, if it drops below another, lower value (some hysteresis should be integrated) the valve is closed again. If a proportional valve is used, it is recommended to rather have a transition range than a transition point to make the change smoother and therefore possibly unperceivable. 


\subsection{Roll compensation and stabilization}

Not all mobile working machines have fully individually suspended and level controlled wheels. In fact, many machines come with no suspension at all or just a preset mechanical suspension (with leaf or coil springs). Some machines have mixed mechanical and hydropneumatic suspensions (front different to rear), some machines have a level controlled (normally hydropneumatic) suspension but only one degree of freedom (vertical) for the whole axle, while the roll is completely fixed - this means its $100 \%$ roll stabilized this way. Some examples of possible setups are show in Fig. 2.

Those machines with individually levelcontrolled wheels can be equipped with the possibility for roll compensation and roll stabilization. This is particularly suitable when strong roll motions can be expected for example due to high COG and / or narrow track width.

Roll compensation is basically the counteraction of the roll movement with the means of the already available valve that also is responsible for the level control / cylinder position control. The valves need to be operated in a way that, for example during cornering, the valve(s) for the outside wheels direct oil into the cylinders, while the valve(s) for the inside wheels release oil from the cylinders.

It is easy to see that, the quicker the changes in roll motion, the more flow must be available to compensate the cylinder position. Therefore, the core of the roll compensation is a very quickly reacting cylinder position adjustment. The quick reaction must be covered both from the hydraulic and from the electronic side and sufficient hydraulic power must be available. The simplest way to achieve this is to have a dedicated, quickened control-loop for the cylinder position, which also uses only the position sensor signal for the respective cylinder. This control loop could then be activated (in addition to the regular position control loop) if certain criteria are fulfilled, for example if steering angle and vehicle speed both exceed certain values.

In a more advanced approach, the activation of the valve is combined with special previewing algorithms, which could for example also use steering angle and speed or the signal from a lateral acceleration sensor. From steering angle and vehicle speed the expected lateral acceleration can be calculated and from this the expected roll angle. With this info, the cylinder can be already filled with oil / released from oil
Front: both wheels suspended together, free oscillation Rear: unsuspended axle, $100 \%$ mech. roll stabilized
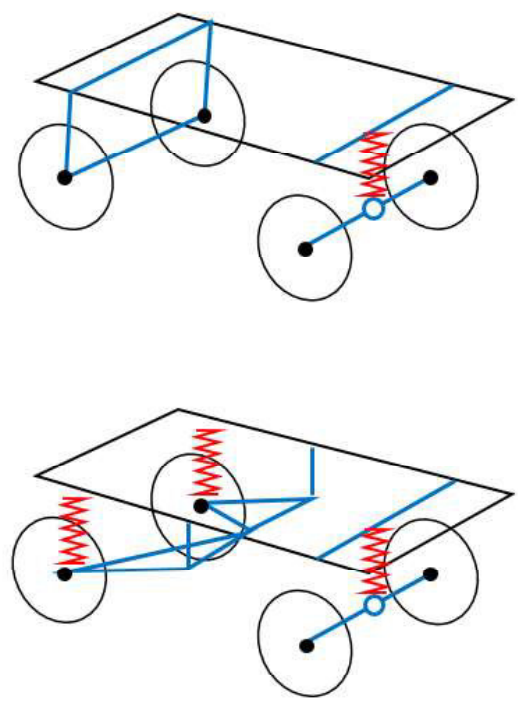

Front: both wheels suspended together, evtl. partially mech. roll stabilized Rear: wheels suspended individually, hydraulically roll stabilized
Front: both wheels suspended together, free oscillation Rear: suspended axle, $100 \%$ mech. roll stabilized
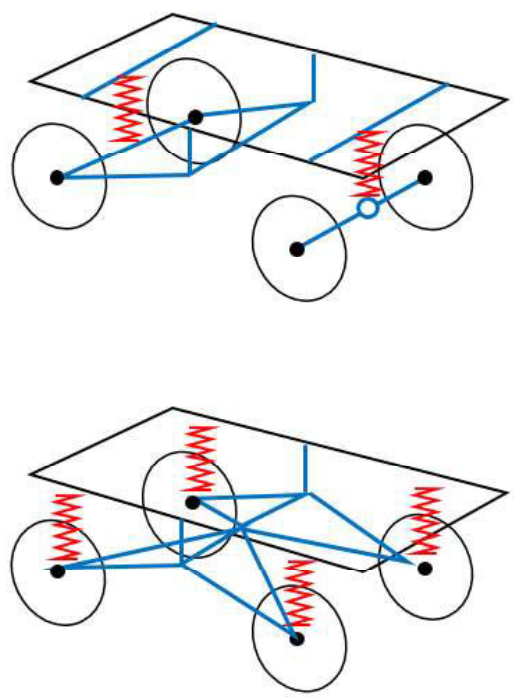

Front: wheels suspended individually, hydraulically roll stabilized Rear: wheels suspended individually, hydraulically roll stabilized

Figure 1: Possible suspension setups for mobile machines 
before this expected roll angle actually is reached. So instead of only reacting (as in the example with the quickened control-loop above) the system can ,pre-act" and thus avoid excessive roll motion.

The abovementioned can also be done, if a sensor is used to directly measure the (lateral) acceleration. Such a sensor offers the additional possibility to do an over-compensation of the expected roll-movement. So during cornering the machine could even lean into the corner (like a motorbike) or, when driving alongside a hill, the machine could be brought upright independent from the slope of the hill.

Compared to roll compensation, which acts rather slowly, a roll stabilization will provide instantaneous counterforces against roll movement right at the moment when the chassis is going to an inclined position. This is achieved by spring forces that act in a direction which try to bring the chassis perpendicular to the ground contact plane. As already mentioned, this can be done with mechanical means (stabilizer bar) or, in case of hydropneumatic suspensions, also with a special hydraulic setup.

This hydraulic setup is using two doubleacting cylinders of a suspended axle that are "cross-connected" to each other. This means the pistonside of the left cylinder is connected to the rodside of the right cylinder and vice versa. In a hydraulic schematic, this is easily visible as a cross-shaped arrangement of hydraulic lines. While, in this setup, the actual load carrying area of the cylinder is the rod diameter, the vertical natural frequency is determined by the accumulator size and precharge. If these parameters are chosen according to the needs of the application, in a next step the roll stiffness can be chosen by a suitable ratio of piston area to rod area. The higher this ratio, the stronger will be the roll stabilization effect.

The very positive effect of hydropneumatic roll stabilization is its inherent adaptation of roll stabilization to the load: the higher the load the higher is also the roll spring rate. This comes from the higher pressure in the cylinders and therefore the compression of the gas volume inside the accumulators. The vertical spring rate rises and with it also the roll spring rate increases. Indeed the latter increases by the power of 2 to the load. For many machines, this has a very positive effect: a linear increase of the roll spring rate with load would be not sufficient, since with increased load, also the COG rises. Both effects, increased load and increased COG, multiply and dramatically change the roll torque during cornering (like with a heavy load on the roof of a car). So the increase with the power of 2 is quite exactly what such machines need for the (ideally) constant roll behaviour independent from the load.

The described roll stabilization is purely based on the hydraulic and mechanical layout of the system with no need for electronic control. However, roll stability is (just as the vertical spring stiffness) also influenced by the switching of accumulators as mentioned above. The tuning of the switching point and the relative size of the switchable accumulator must therefore consider both the vertical and the roll behaviour of the suspension. This means the test procedure must include pure straight driving on uneven terrain and also fast cornering in different loading conditions as well as the transition from a straight into a curve (needed to tune roll damping).

\subsection{Pitch compensation and stabilization}

The same principles apply as for the roll motion. Some machines even use the same ,crossconnected" setup for pitch stabilization as described above for roll stabilization. Fortunately, the wheelbase of a machine is typically significantly higher than its track width, so usually the need for additional pitch stabilization is not very urgent. Normally the already available control for cylinder position is sufficient to compensate long-term pitch movements caused for example by weight shifts by driving steep uphill or downhill for a longer time.

For short-term pitch movements, coming for example from heavy braking, it is also possible to quickly increase the damping or quickly increase the spring rate by switching a second accumulator off during the period of deceleration (as described above). By designing a suitable mechanical setup of the suspension, for example a leading link design, the pitch movements can already be significantly reduced just based on mechanics. However, certain boundary conditions (for example design space) do not always allow such a setup. 


\subsection{Equalization of tyre-to-ground forces}

Ensuring optimal ttg forces has several advantages. First of all, it enables best possible traction and therefore driving safety as well as efficiency. Secondly, an unsuitable distribution of ttg forces also spoils comfort, since the stronger loaded wheels will create higher vertical natural frequencies, while the less loaded wheels are suspended too soft and therefore feel spongy. This way, the ride behaviour becomes less comfortable and less predictable. In extreme cases, it could even lead to tipping over of the vehicle.

In a four-wheel / four-corner suspension, an unsuitable distribution off ttg forces typically comes from the hyperstatic character of this setup. Normally three wheels / contact points to the ground would make the system isostatic and thus only one distribution of $\mathrm{ttg}$ forces is possible. However, with the fourth wheel, there is one contact point too much so it is unclear how the vehicle weight will be transferred to the ground. Everybody knows the table (or chair) with four legs that rocks in a certain way only because one leg is slightly too short/long or because the ground is not completely even. The table rocks along the axis of the two legs that are slightly longer and therefore need to take most of the load. Indeed sometimes these two legs take all the weight from the table while the other two legs are almost unloaded.

The same applies to a vehicle with four wheels, one in each corner. If the suspension position of each of these wheels is not in exactly the right position, the weight distribution will be unsuitable. Unsuitable in this case means that the weight distribution is not as it would be expected to be ideally due to the COG, the mechanics and the geometry. This is something very important to consider, since in a 4-wheeled vehicle, in almost no condition will the $\mathrm{ttg}$ forces at each wheel be a quarter of the weight. For this case the COG would have to be in the center of the vehicle, it would have to be driving on perfectly horizontal ground and with no lateral and no foraft accelerations. That is nearly never the case and that's why ttg forces of all the wheels can almost never be exactly equal.

The topic of ttg force equalization is important for the design of suspensions already for many decades. In the beginning, it was only considered for sports cars, who had to have, for best traction, optimal ttg force distribution. The ttg force distribution was manually set using a wheel force scale. Only once the automatic level-readjusting suspensions started to become more important, the ttg forces moved into the foreground of general development. Most work has been done in the area of passenger cars, which typically use pneumatic suspensions. Some work was also based on hydraulics, however often focused on active suspension systems. While in the beginning, the ttg force calculation could be done only during standstill or stationary straight driving [3], later these conditions were not necessary anymore ([4] and [5]) and the readjustment of $\mathrm{ttg}$ forces could be done at any driving condition.

Normally, the first step in such an approach is, to calculate the ideal distribution of ttg forces under the given circumstances. To do that, it is necessary to find the (virtual) point of intersection of the vehicles COG gravity vector with the contact plane of the four wheels. The position of this point then allows to calculate for each wheel the ideal load under these circumstances.

The next step then is, to measure the actual load on each wheel. The deviation of the actual load from the ideal load then allows to calculate corrective actions. Fortunately, in hydropneumatic suspensions, there is an easy source of information for the calculation. A pressure sensor in each suspension cylinder (at each wheel) gives the information about the current situation, from which also the ideal load distribution can be deduced. Indeed the cylinder pressures do not perfectly represent the ttg load (weight of the unsprung masses is not considered, friction inside the cylinders) but in tests they proven to provide a sufficiently accurate information representative for the ttg load.

The corrective actions can be taken for each wheel individually, however for simplification, ARGO-HYTOS chose to group the wheels in diagonals [6]. Diagonal 1 is the front left wheel and the rear right wheel, while diagonal 2 is the front right wheel and the rear left wheel. If a corrective action is done, it is always applied to both wheels of a diagonal. For safety reasons, only the diagonal, where the load is higher than ideally calculated, is corrected by relieving oil from these cylinders. This way the pressure in these cylinders drops, these cylinders contract slightly and thus transfer more weight to the other 


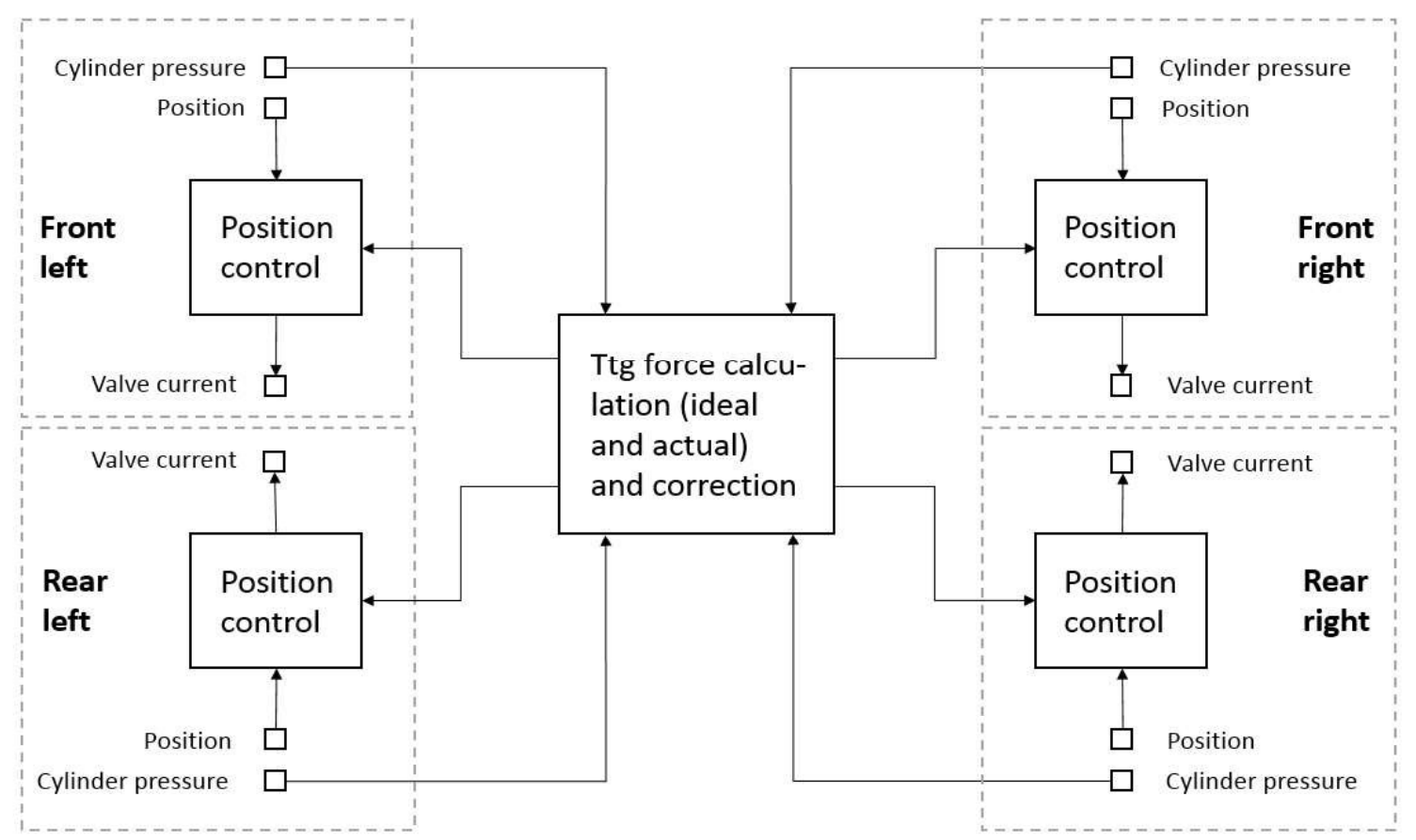

Figure 3: Control schematic for tyre-to-ground force optimization

diagonal. Once the load deviation of the higher loaded diagonal is again inside a tolerance band, the corrective action is ended. By lowering the higher loaded diagonal, it is ensured that at no time the cylinders are extended by the diagonal control loop, which would cause a lifting of the $\mathrm{COG}$ and therefore could bring the machine in a critical tip-over condition.

It is obvious, that this control loop interferes with the position control loop of each wheel, which is supposed to keep the vehicle in a defined position. ARGO-HYTOS decided to melt the diagonal control into the position control loop and in the end only get one output signal for the proportional valve that controls the position (Fig. 3). To enable this, the diagonal control loop weight deviation is converted into a "virtual“ position deviation. The latter is then added to the actual position deviation of each of the wheels of the respective diagonal (actual position - set position). This signal is then fed into the regular position control loop, where the normal, state-ofthe-art position control procedures are applied. The advantage of melting both loops into one control is firstly simplification and secondly also the right compromise between correct cylinder position and best ttg force distribution. For customization, this compromise can be shifted towards either one or the other goal by changing the parameterization of the algorithm.

A useful by-product of the aforementioned calculations is the information about the available traction on each wheel as well as the information about how close the machine is to tipping over. These informations are sent to the main machine controller so the OEM can decide himself how to consider this info in his own algorithms.

The described algorithms have proven their efficacy on several all-wheel suspended machines already, among them self-propelled sprayers, a self-propelled feed-mixer and a road sweeper.

\section{APPLICATION EXAMPLE}

The realization of the above-mentioned methods is shown in the application on a self-propelled sprayer. All wheels are individually suspended while the wheels of an axle are cross-connected for roll stabilization. The schematic for front and rear axle are identical, that's why only the schematic for the front axle is shown in Fig. 4.

Due to the high tank capacity of 50001 and a $36 \mathrm{~m}$ boom, the load ratio on the hydraulic suspension cylinders is about $3: 1$ (max:min). For example, the highest load on the front axle appears with full tank and folded boom, while the 


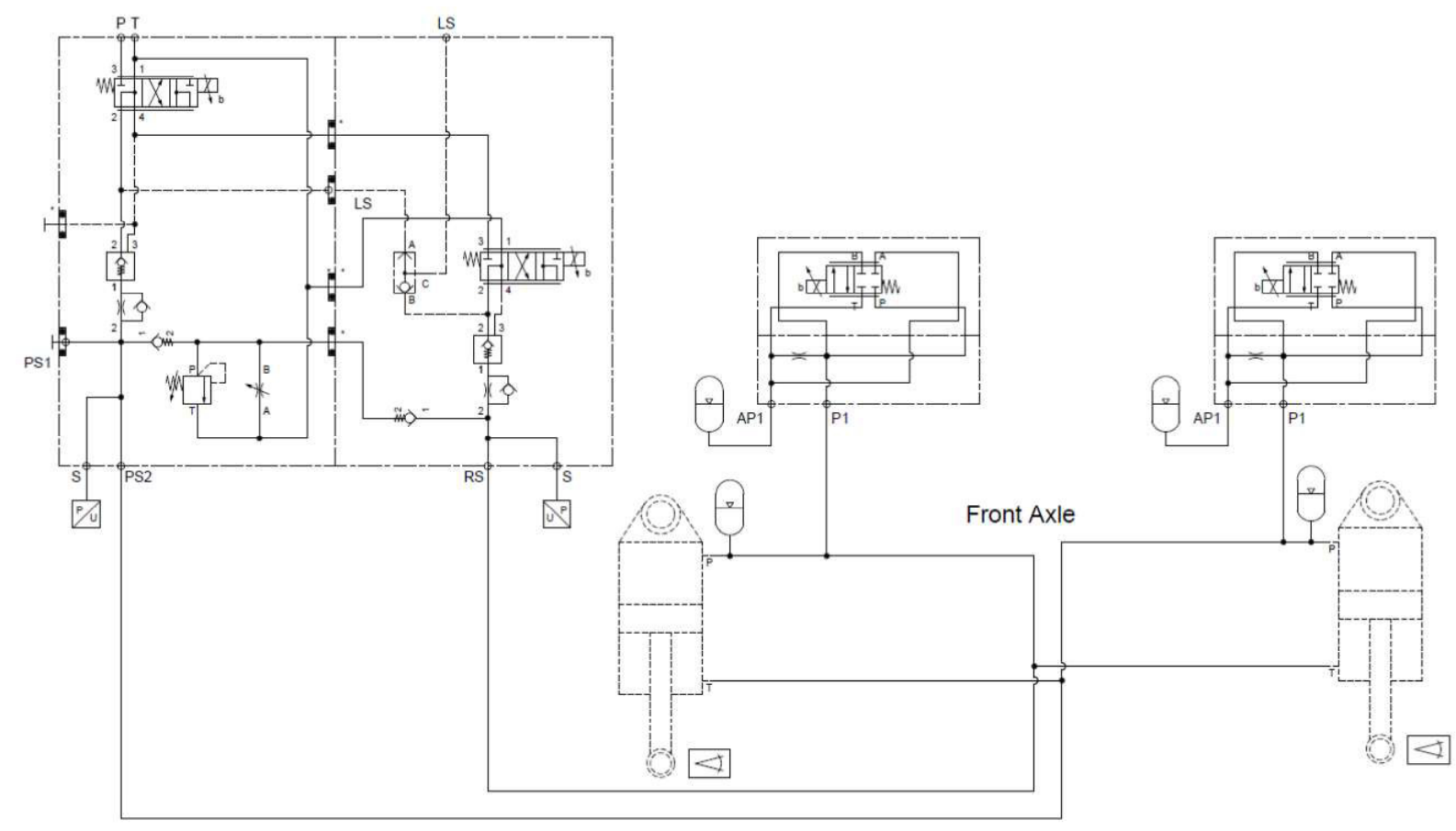

Figure 4: Hydraulic schematic for the front axle of an all-wheel suspended self-propelled sprayer

lowest results from empty tank and boom deployed. Due to the high load ratio, a simple unpreloaded cylinder+accumulator-setup was not possible. Instead, the switching of a second accumulator was implemented as already described in section 3. For this purpose, a standard 4/2-proportional Cetop valve is integrated in ,double-flow" condition, so both metering edges are used for the connection between cylinder and accumulator, which offers very low pressure drop when fully opened. A bypass orifice between accumulator 1 and accumulator 2 ensures permanent pressure equalization of accumulator 2 even if the proportional valve is fully closed.

The hydraulic level control is done based on ARGO-HYTOS special 4/3-prop-levelling valve (25 lpm-version). For each suspension cylinder, one valve is needed. Each of these valves uses only one solenoid, which keeps the number of required PWM-outputs of the control unit low. The input signal for the level control comes from a position sensor (angle sensor). As mentioned in section 3 , pressure sensors are attached to each level control circuit to allow to calculate and adjust the right ttg forces for each wheel.

The control is done by one control unit each for the front and the rear axle. With a CANconnection in between, the front control unit is the master and also contains the central logics. Both control units read four sensor signals and operate four PWM outputs.

\section{CONCLUSION AND OUTLOOK}

For maximum comfort, driving safety and best working results, a hydropneumatic all-wheelsuspension offers many advantages over other types of systems. It automatically adapts its rolland pitch-stiffness to the loading conditions and also allows, based on a dedicated control loop, to permanently measure, calculate and readjust the tyre-to-ground forces to the current operating conditions. Quick level adjustment, with high hydraulic power, can help to quickly adapt to load changes or to compensate roll and pitch movement when driving in hilly terrain. Hydropneumatic all-wheel suspensions are a well-proven technology in many different applications already. Since they fulfil the growing demand for comfort, working performance and efficiency, they are likely to be used in even more machine types in the future. 


\section{REFERENCES}

[1] Bauer, W.; Mejsnar, P.; Englberth, E.; Fischer, M.: 4/3 proportional valve with only one solenoid: A new technology for position control in suspension systems, 9th IFK, March 24-26, 2014, Aachen, Germany

[2] Bauer, W.: Hydropneumatic suspension systems, Springer Verlag, Berlin, Heidelberg, 2011

[3] DE4003188, Verfahren zur Steuerung der Höhe eines Fahrzeuges, Fuji Jukogyo K.K., Tokyo, JP

[4] DE4323544, Aufhängungssystem für Kraftfahrzeuge, Mazda Motor Corp and Naldec Corp, both of Hiroshima, JP

[5] DE10244363, Verfahren zur Regelung und/oder Steuerung eines aktiven und/oder steuerbaren Fahrwerks, DaimlerChrysler AG, Stuttgart, DE

[6] DE102018118062, Verfahren und System zur Lageregelung eines Fahrzeuges, FSP Fluid Systems Partners Holding AG, Baar, CH 